\title{
Educação em Saúde para Idosos: uma revisão bibliométrica da produção científica
}

\section{de 2017 a 2021}

\author{
Health Education for the Elderly: a bibliometric review of scientific production from 2017 to 2021 \\ Educación para la salud de las personas mayores: revisión bibliométrica de la producción científica
}

de 2017 a 2021

\section{Resumo}

Contexto: A educação em saúde é um método preventivo eficaz para apoiar os cuidados necessários para uma vida plena. Muitos cuidados são necessários na idade idosa, tendo em vista a vulnerabilidade desta população. Objetivo: apresentar o perfil da produção científica sobre os temas Educação em Saúde ligado à população idosa entre os anos de 2017 e 2021. Metodologia: Foi realizada uma revisão bibliométrica, com buscas nas bases de dados Web of Science e Scopus, utilizando os descritores Educação em saúde, Educação para saúde e idoso, com seus resultados tratados pelo software RStudio. Resultados: Foram encontrados 345 documentos, 1328 autores, EUA, Espanha, Reino Unido, China e Brasil foram os 5 melhores países no ranking de produção de estudos, no ranking de revistas, o melhor foi EUA, seguido do Reino Unido (com 3 posições no ranking) e Turquia. Entre as 10 maiores instituições de pesquisa, 9 são universidades. O descritor mais usado foi Health Education. Houve evolução nos descritores de faixa etária de jovens adultos para meia idade e um aumento de 404,54\% no crescimento de publicações sobre o tema nos últimos 5 anos. Considerações finais: O perfil da produção científica ainda é pouco expressivo mundialmente, dada a importância do tema nas esferas econômica e social. Os países com maior população idosa, não aparecerem nos principais rankings de publicações. O Brasil figura em $5^{\circ}$ lugar na produção autoral sobre o tema. O financiamento de pesquisa está principalmente em universidades. Os idosos ainda não estão devidamente representados pelos descritores.

Palavras-chave: Educação em saúde; Educação; Idoso; Bibliometria.

\begin{abstract}
Context: Health education is an effective preventive method to support the care needed for a full life. Much care is needed in the elderly, given the vulnerability of this population. Objective: to present the profile of scientific production on Health Education topics related to the elderly population between the years 2017 and 2021. Methodology: A bibliometric review was carried out, with searches on the Web of Science and Scopus databases, using the descriptors Education in health, Education for health and the Elderly, with their results treated by the RStudio software. Results: 345 documents were found, 1328 authors, USA, Spain, UK, China and Brazil were the 5 best countries in the ranking of production of studies, in the ranking of journals, the best was USA, followed by the UK (with 3 positions in the ranking) and Turkey. Among the top 10 research institutions, 9 are universities. The most used descriptor was Health Education. There was an evolution in the age group descriptors from young adults to middle age and an increase of $404.54 \%$ in the growth of publications on the subject in the last 5 years. Final considerations: The profile of scientific production is still not very expressive worldwide, given the importance of the topic in the economic and social spheres. Countries with the largest elderly population do not appear in the main rankings of publications. Brazil ranks 5th in the authorial production on the subject. Research funding is primarily at universities. The elderly are still not properly represented by the descriptors.
\end{abstract}

Keywords: Health education; Education; Older adult; Bibliometrics. 


\section{Resumen}

Contexto: La educación en salud es un método preventivo eficaz para apoyar los cuidados necesarios para una vida plena. Se necesita mucho cuidado en los ancianos, dada la vulnerabilidad de esta población. Objetivo: presentar el perfil de la producción científica sobre temas de Educación en Salud relacionados con la población anciana entre los años 2017 y 2021. Metodología: Se realizó una revisión bibliométrica, con búsquedas en las bases de datos Web of Science y Scopus, utilizando los descriptores Educación en salud, Educación para la salud y Adulto Mayor, con sus resultados tratados por el software RStudio. Resultados: se encontraron 345 documentos, 1328 autores, USA, España, UK, China y Brasil fueron los 5 mejores países en el ranking de producción de estudios, en el ranking de revistas el mejor fue USA, seguido de UK (con 3 posiciones en el ranking) y Turquía. Entre las 10 principales instituciones de investigación, 9 son universidades. El descriptor más utilizado fue Educación en Salud. Hubo una evolución en los descriptores de grupos de edad de adultos jóvenes a mediana edad y un aumento del 404,54\% en el crecimiento de las publicaciones sobre el tema en los últimos 5 años. Consideraciones finales: El perfil de la producción científica aún no es muy expresivo a nivel mundial, dada la importancia del tema en el ámbito económico y social. Los países con mayor población anciana no aparecen en los principales rankings de publicaciones. Brasil ocupa el quinto lugar en producción autoral sobre el tema. La financiación de la investigación se encuentra principalmente en las universidades. Los ancianos aún no están debidamente representados por los descriptores.

Palabras clave: Educación para la salud; Educación; Anciano; Bibliometría.

\section{Introdução}

A educação em saúde é um tema de suma importância na prevenção de doenças, sejam estas físicas ou psicológicas e na manutenção de uma vida saudável ao longo da trajetória de envelhecimento do ser humano. Conhecer os principais enfoques, os principais grupos de pesquisadores e a distribuição geográfica da sua produção científica pode ajudar a agregar conhecimentos na construção de um saber consolidado sobre a saúde e sua abrangência na idade idosa, além de possibilitar o desenvolvimento de políticas públicas. Este trabalho de construção do conhecimento pode ser apoiado por revisões bibliométricas, de escopo e sistemáticas.

Mas o que é a educação em saúde? Segundo a informação do vocabulário estruturado e multilíngue da base de dados BVS para descritores em saúde (DeCS) (Bireme, 2021), as composições das palavras 'educação' e 'saúde', compõe os descritores: 'educação e saúde', 'educação em saúde' e 'educação para a saúde', que são citados como sinônimos no mesmo descritor 'educação na saúde'.

Ainda nesta consulta ao DeCS, o escopo para a educação em saúde foi definido como “A educação em saúde objetiva desenvolver nas pessoas um sentido de responsabilidade, como indivíduo, membro de uma família e de uma comunidade, para com a saúde, tanto individual como coletivamente" (Bireme, 2021, p. [online]).

De acordo com o glossário eletrônico da BVS (Brasil, 2021), a 'educação na saúde' consiste na produção e sistematização de conhecimentos relativos a formação e ao desenvolvimento para atuação em saúde, envolvendo práticas de ensino, diretrizes didáticas e orientação curricular e a 'educação em saúde' é "um processo que envolve a capacitação de pacientes, cuidadores e profissionais de saúde, estimulando-os a agir conscientemente diante de cada ação do cotidiano, criando um espaço para o aprimoramento de novos conhecimentos e práticas” (Brasil, 2021, p. [online])

A importância de saber sobre educação em saúde para idosos reside na preocupação de que esta população encontra diversos desafios que lhes são próprios desta etapa da vida, questões sobre como ocorre o envelhecimento biológico do corpo humano, quais devem ser as condutas para um envelhecimento bem-sucedido, dos desafios psíquicos relacionados a autopercepções, como a autoeficácia, autoimagem, e como estas questões psicológicas afetam o bem estar, a longevidade com qualidade de vida, além de desafios sociais, agravados por normopatias da velhice, sejam estas ocorrendo em espaços intrafamiliares, como externos, considerando aspectos inter-relacionais. Como atualmente esta etapa da vida tem se mostrado muito mais longa do que a infância, adolescências e juventude juntas, há de haver meios para que este percurso seja ao mínimo agradável, que proporcione perspectivas futuras e possibilidades de realização pessoal, criação de metas de vida e de saúde (Vagetti et al., 2020). 
Esta revisão bibliométrica buscou resolver a seguinte questão norteadora: Como se apresentou a produção científica sobre o tema Educação em Saúde para Idosos entre os anos de 2017 e 2021? O objetivo geral deste estudo foi apresentar o perfil da produção científica sobre os temas Educação em Saúde ligado à população idosa entre os anos de 2017 e 2021, e por objetivos específicos: analisar a rede de colaboradores com os critérios da lei de Lotka; apresentar a dispersão dos documentos com o uso da lei de Bradford e, demonstrar a frequência de descritores utilizados nas publicações, conforme a lei de Zipf.

\section{Metodologia}

Este estudo trata-se de uma revisão bibliométrica (Soares et al., 2018), de caráter exploratório e de abordagem quantitativa. As pesquisas do tipo bibliométrica são caracterizadas por apresentarem um caráter exploratório, dada a busca pela produção científica mundial sobre determinado tema, elaborando os índices de produção, das relações de cooperação na produção científica entre autores e países, além de abordar a forma de disseminação do conhecimento e suas possíveis lacunas (Mugnaini et al., 2017).

\subsection{Produção dos Dados}

Foram feitas buscas nas bases de dados Web of Science e Scopus, para o tema Educação em Saúde e Idosos, utilizandose por padrão a pergunta (query) da frase de pesquisa (string): (("educação em saúde" OR "health education" OR "educação para saúde" OR "educación en salud") AND (idoso OR aged OR anciano OR elder OR elderly OR "older adults"), que foi aplicada em ambas as bases. Estas bases foram eleitas por serem mais abrangentes e aceitas na comunidade científica (Pérez-Escoda, 2017), além de permitirem a mineração e exportação dos dados de forma a facilitar a exploração dos mesmos com o uso de softwares.

A busca, extração e análise dos dados para este estudo foi desenvolvida, principalmente, a partir dos pressupostos das três teorias fundamentais da Bibliometria, que são: a lei de Lotka, que propõe analisar o comportamento da rede de autores (authors); a lei de Bradford, que faz a representação da dispersão das fontes (sources) e, por fim, a lei de Zipf, que demonstra as frequências das coocorrências das palavras chave (keywords) (Soares et al., 2018).

\subsection{Critérios de elegibilidade}

Foram incluídos os documentos que possuíram acesso aberto nas bases de dados, que continham nos títulos, resumo, ou palavras-chave o tema Educação em Saúde e Idosos, considerando os anos de publicação de 2017 a 2021.

Como critérios de exclusão, não foram considerados os documentos que não tratem de educação em saúde e idosos, que estejam foram do período de tempo pesquisado, que tratam de outras faixas etárias e que não possuam o acesso aberto para leitura.

\subsection{Análise, tratamento e apresentação dos dados}

Os dados coletados nas bases foram exportados no formato de referências (Bibtex) e importados pelo software RStudio $^{l}$ (Rstudio, 2022), sendo feita a unificação dos resultados e uma única base de dados (database). Foram utilizados os pacotes do RStudio para elaboração de bibliometrias chamados Bibliometrix e o Shiny UI for bibliometrix package (biblioshiny) necessários para a extração das análises, grafos e dados importantes para este estudo (Aria \& Cuccurullo, 2021) e o software Ms Excel® 2013 para a elaboração de tabelas e quadros. Os resultados foram apresentados em tabelas, quadros e figuras, que demonstraram as análises e recortes por temática.

\footnotetext{
${ }^{1}$ RStudio Version 1.1.463, interface de R version 4.0.3 (2020-10-10) -- "Bunny-Wunnies Freak Out" Copyright (C) 2020 The R Foundation for Statistical Computing Platform: i386-w64-mingw32/i386 (32-bit)
} 


\subsection{Aspectos Éticos e Legais}

Este estudo seguiu as orientações do Committèe on Publication Ethics (Cope, 1999), que estabelece as boas práticas e informações para autores e editores sobre a ética em pesquisa e seu protocolo foi registrado na base científica Open Science Framework (OSF) (Flores-Gomes et al., 2021).

\section{Resultados e Discussão}

As buscas nas bases de dados resultaram em 346 artigos, sendo 265 trabalhos originados na Web of Science e 81 trabalhos na base Scopus. Destes achados foi excluído um documento por duplicidade, restando 345 trabalhos eleitos para esta revisão, conforme apresentado na Tabela 1.

Tabela 1. Informações do cluster de documentos coletados nas bases de dados.

\begin{tabular}{lr}
\hline \multicolumn{2}{c}{ Principais informações sobre dados } \\
\hline Descrição & Resultados \\
\hline Intervalo de tempo & $2017: 2021$ \\
Fontes (revistas, livros, etc) & 141 \\
Documentos & 345 \\
Média de anos de publicação & 2,22 \\
Média de citações por documentos & 2,948 \\
Média de Citações por ano por documento & 0,8302 \\
Referências utilizadas & 13258 \\
\hline
\end{tabular}

Fonte: Autores (2022).

Ainda que seja expressiva a quantidade de fontes, não foram muitos os documentos eleitos nesta revisão. A razão encontrada de 2,44 documentos por periódico, em um período de 5 anos, é um número relativamente pequeno para um tema de tal importância. A média de referências por documento foi de 38,42 estudos e pode-se perceber que os estudos produzidos sobre o tema foram citados menos de uma vez por ano. Estes dados em si já apresentam uma preocupação com o desenvolvimento do assunto no meio acadêmico.

Na Tabela 2 estão estratificados os tipos de documentos encontrados, sendo na sua maioria artigos originais já publicados. As buscas não foram limitadas apenas a artigos, mas buscou-se todos os documentos disponíveis sobre o tema, para que os achados tornem o tema mais amplo possível. 
Tabela 2. Tipos de documento resultantes das buscas.

\begin{tabular}{lr}
\hline \multicolumn{2}{c}{ Tipos de documento } \\
\hline Descrição & Resultados \\
\hline Artigos & 276 \\
Artigos; acesso antecipado & 7 \\
Artigos; papers em processo & 3 \\
Revisão do livro & 1 \\
Paper de conferência & 1 \\
Editorial & 5 \\
Outros papers em processo & 33 \\
Revisões & 19 \\
\hline Total & $\mathbf{3 4 5}$ \\
\hline
\end{tabular}

Fonte: Autores (2022).

Os achados sugerem que o estudo deste tema é recente nas bases consultadas, dada a importância e quantidade de documentos encontrados, o que possibilita a inferência de que o tema não tenha ainda a maturidade necessária para despertar uma maior abordagem no meio científico, ou a educação em saúde e idosos ainda não seja um tema de interesse da comunidade acadêmica, ainda que o tema do envelhecimento tenha sido discutido nos últimos anos, principalmente em face do crescimento demográfico desta população (idosos).

Outra observação é que dos 345 documentos encontrados, apenas 276 são artigos completos, o que também caracteriza como o tema foi estudado neste período. Pode-se inferir que, ainda que o tem em si seja de interesse, talvez fatores de maior urgência, como estudos sobre a pandemia de COVID-19, tenham conquistado maior relevância frente às questões do ensino sobre saúde no envelhecer.

Em conferências, que é o local de disseminação do conhecimento mais efetivo entre os profissionais da área, apenas 1 documento foi encontrado nas bases de dados pesquisadas, no período de 5 anos, que revela também pouco investimento na busca de soluções educativas para idosos e profissionais de educação e saúde.

A educação em saúde pode ajudar a reduzir os custos na área de saúde, melhorando assim o processo de investimento em pesquisa, em profissionais, em instalações e equipamentos (Saldiva \& Veras, 2018). Esta educação pode também ser desenvolvida no processo de atenção primária em saúde, proporcionando melhor qualidade de vida aos idosos, aumento da sua expectativa de vida e de sua longevidade efetiva. Em geral, o conhecimento sobre como conduzir seu estilo de vida quando ainda se é jovem, proporciona ganhos futuros, com melhor condução dos processos de envelhecimento e reduzir os efeitos deletérios próprios da velhice. Dadas as circunstâncias dos achados desta busca, a falta de mais estudos em educação para a saúde pode ser considerada uma lacuna para produção de estudos futuros.

Nos próximos tópicos serão exploradas as leis fundamentais da Bibliometria, para que os dados sejam apresentados de forma sistematizada, a partir do nível de análise de autores (Lei de Lotka), análise das fontes de documentos (Lei de Bradford) e por fim o detalhamento das palavras-chave (descritores) utilizados nos documentos (lei de Zipf).

Os indicadores bibliométricos, em geral, podem ser subdivididos em quatro abordagens específicas, a saber: “1) qualidade científica; 2) atividade científica; 3) impacto científico; e 4) associações temáticas” (Beira et al., 2020, p. 387). Estes indicadores podem revelar diferentes aspectos da ciência, sejam estes aspectos relacionados a políticas de produtividade de estudos (artigos), nos efeitos do investimento e fomentos às atividades de pesquisa e também, da gerência destes recursos nos processos de investigação e desenvolvimento de ciência. 
Os indicadores bibliométricos são utilizados, particularmente, como ferramentas ou instrumentos úteis para a avaliação em áreas específicas da cientometria, na produção de estudos quantitativos que exploram aspectos métricos de conjuntos de dados bibliográficos e linguísticos, extraídos das bases de dados eletrônicas (Mugnaini et al., 2017).

\subsection{Leí de Lotka (authors)}

Criada em 1926, a Lei de Lotka propõe que a produtividade de autores está definida pela Lei do Inverso do Quadrado $\left(1 / n^{2}\right)$ ou " $y_{x}=6 / p^{2} x^{2}$, onde $y_{x}$ é a frequência de autores publicando número $x$ de trabalhos e a é um valor constante para cada campo científico", que pode ser reduzido para a equação y $=$ c.x ${ }^{-2}$ (Araújo, 2006, p. 40).

Segundo Lotka, a produção dos autores que mais publicam sobre um determinado assunto é semelhante à produção dos autores que produzem pouco, ainda que os que produzem pouco sejam a grande maioria dos autores (Silva \& Mayworm, 2020). Este indicador bibliométrico possibilita identificar se a concentração da produção científica, sobre determinado tema, ocorre em torno de um pequeno número de autores, distinguindo quais são estes principais autores e quais são os autores eventuais. (Gregorio-Chaviano et al., 2020).

A Figura 1 demonstra que a linha de tendência de valores esperados (valores projetados) tem expectativas menores do que a observada na linha de tendência observada (número realizado de autores), o que denota que o tema tem uma maior produtividade e vem ganhando força por parte dos autores, além do o esperado. $\mathrm{O} \mathrm{R}^{2}$ do modelo explica $97,59 \%$ da variabilidade dos resultados em seus pontos médios.

Este gráfico foi elaborado eletronicamente pela ferramenta Biblioshiny e demonstra que há um grande número de autores que publicam um único documento e que 7 autores publicaram mais que 3 vezes (Lotka $=1 /(1379)^{2}=5,25 \equiv 7$ ). A tabela 3 demonstra este detalhamento da produção, considerando os 345 documentos recuperados na busca nas bases de dados.

Figura 1. Gráfico de dispersão da produção científica sobre educação em saúde para idosos, equação de Lotka, valores observados e esperados.

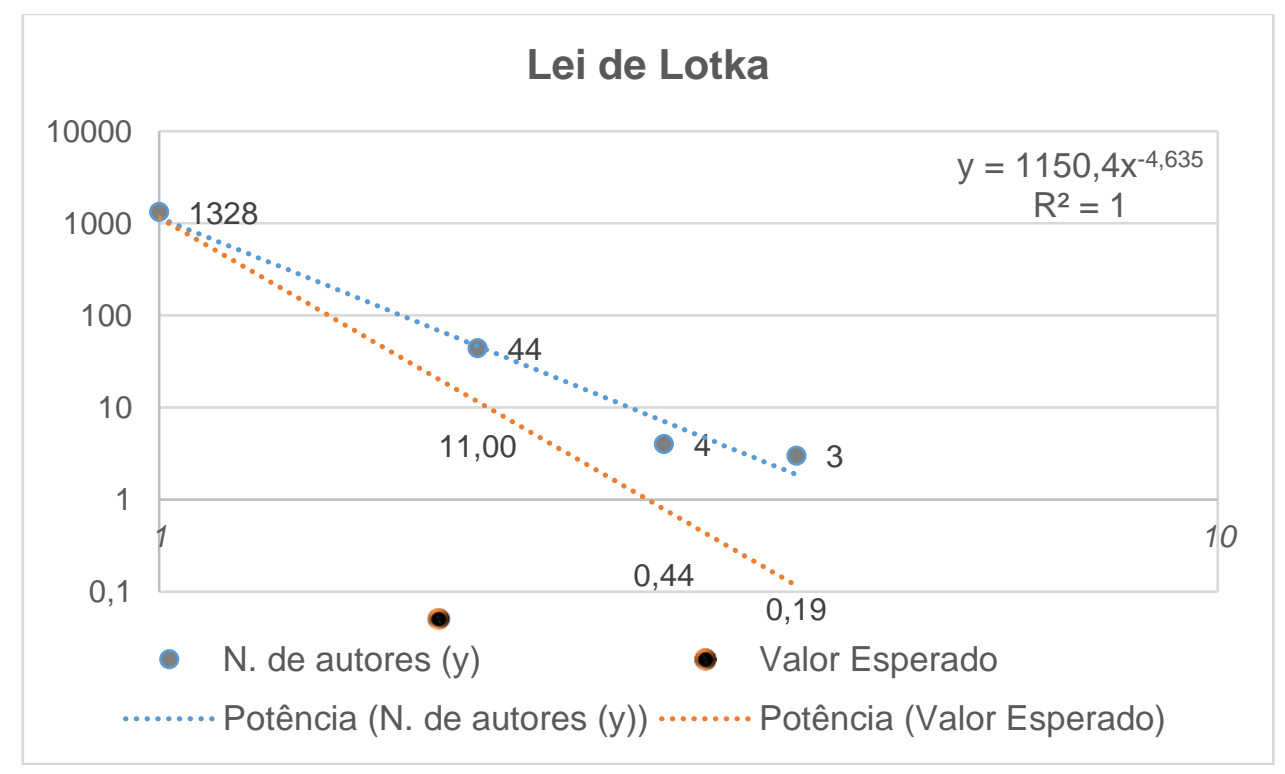

Fonte: Autores (2022).

Na Figura 1 está representada a produtividade encontrada nas buscas realizadas nas bases Web of Science e Scopus sobre o tema Educação em Saúde e idosos. 
Tabela 3. Produção de autores, por número de documentos produzidos.

\begin{tabular}{crrr}
\hline Documentos escritos $(\mathbf{x})$ & $\mathbf{N}^{\circ}$ de autores $(\mathbf{y})$ & Valor Esperado & Proporção de Autores \\
\hline 1 & 1328 & 1328 & $96,30 \%$ \\
2 & 44 & 11 & $3,19 \%$ \\
3 & 4 & 0 & $0,29 \%$ \\
4 & 3 & 0 & $0,22 \%$ \\
\hline Total de autores & 1379 & 1339 & $100,00 \%$ \\
\hline
\end{tabular}

Fonte: Autores (2022).

Neste caso, o resultado de 1328 autores com 1 documento considerou as coautorias nos estudos, que em alguns casos pode passar de 10 autores por documento. No caso de estudos sobre a infecção por COVID-19, alguns artigos possuíam 25 autores. Para as demais faixas, percebe-se que o número menor é menos disperso, em que se considera que menos autores compartilham de mais estudos em comum.

Um outro indicador a se considerar é o de colaboração entre autores. A Tabela 4 apresenta o índice de colaboração entre os autores, que representa a atividade de produção científica, sendo que este indicador retornou a média de 4,38 autores por documento para este tipo de associação temática. Segundo Gregorio-Chaviano, Limaymanta e López-Mesa (2020), os padrões de colaboração estão diretamente ligados ao relacionamento entre os diversos autores, sejam em laboratórios/grupos de pesquisa, como por afinidade ou ainda pelo formato do processo de escrita, que pode ser fomentado por empresas públicas ou privadas interessadas em pesquisa, e abrangência da participação multifatorial do tema pesquisado.

Tabela 4. Dados informativos da colaboração entre autores.

\begin{tabular}{ll}
\hline Colaboração entre autores & \\
\hline Descrição & Resultados \\
\hline Documentos com único autor & 38,00 \\
Média de documentos por autor & 0,25 \\
Média de autores por documento & 4,00 \\
Média de coautores por documento & 4,17 \\
Índice de colaboração & 4,38 \\
\hline
\end{tabular}

Fonte: Autores (2022).

Os dados da Tabela 4, em comparação com dados da tabela 3, é possível verificar que os 1328 autores com um único documento não escreveram estes documentos sozinhos, tendo em vista que apenas 38 documentos apresentaram 1 único autor (Tabela 4). O indicador de média de documentos por autor traz um resultado de pesquisa referente ao período de 5 anos, o que sugere que a produção de estudos ocorre, em média, a cada 2 anos aproximadamente.

A colaboração entre autores pode ser realizada interna ou externamente aos países de origem. Na tabela 5 estão descritos os tipos de colaboração que ocorreram na amostra pesquisada. Os Estados Unidos detêm a maior colaboração interna entre autores, com um índice de colaboração interna de 42 pontos, enquanto o Brasil figura na $5^{\mathrm{a}}$ posição, com 24 pontos para este mesmo indicador. 
Tabela 5. País do autor correspondente, indicadores de colaboração interna e externa entre autores, considerando os 10 países com maior participação na produção de documentos.

\begin{tabular}{llllll}
\hline País & Documentos & Freq. & SCP & MCP & MCP_Ratio \\
\hline Usa & 45 & $14 \%$ & 42 & 3 & $7 \%$ \\
Spain & 34 & $10 \%$ & 33 & 1 & $3 \%$ \\
United kingdom & 30 & $9 \%$ & 24 & 6 & $20 \%$ \\
China & 26 & $8 \%$ & 23 & 3 & $12 \%$ \\
Brazil & 25 & $8 \%$ & 24 & 1 & $4 \%$ \\
Australia & 16 & $5 \%$ & 15 & 1 & $6 \%$ \\
Canada & 10 & $3 \%$ & 8 & 2 & $20 \%$ \\
Ethiopia & 8 & $2 \%$ & 8 & 0 & $0 \%$ \\
Sweden & 7 & $2 \%$ & 6 & 1 & $14 \%$ \\
Iran & 6 & $2 \%$ & 5 & 1 & $17 \%$ \\
\hline
\end{tabular}

Nota. Freq: frequência relativa da produção de documentos pelo país; SCP: índice de colaboração interna de autores do país; MCP: índice de colaboração de autores entre países; MCP_Ratio : frequência relativa da participação externa do país. Fonte: Autores (2022).

Quando considerada a colaboração entre os autores de outros países, o Reino Unido lidera com um indicador de 6 pontos, o dobro do indicador apresentado pelos Estados Unidos e 6 vezes maior que o indicador brasileiro.

Esta colaboração internacional pode acrescentar consistência metodológica e maior extrapolação de resultados, o que contribui para o crescimento do conhecimento sobre o tema. Neste caso, o Brasil estaria figurando em $6^{\circ}$ lugar, em números absolutos, e em $8^{\circ}$ lugar na frequência relativa [MCP_RATIO = (MCP/Documentos) 100$]$. O indicador de colaboração internacional também demonstra uma reserva de mercado nacional sobre as pesquisas científicas, o que diminui a velocidade do progresso científico. Países mais desenvolvidos apresentam um índice de colaboração maior que países em desenvolvimento ou subdesenvolvidos, e a conexão entre estes mundos fornece ajuda mútua na compreensão dos fenômenos.

A Figura 2 ilustra a força das ligações colaborativas entre países, sendo que quanto maior for a espessura da ligação, mais forte foi esta interação entre os coautores. Estas ligações são importantes para a análise, pois "as características dos processos de internacionalização materializam nas publicações diferentes ações que determinam a amplitude de uma rede" (Gama et al., 2021, p. 14). Visualmente, a figura 2 mostra os países com maior produção colaborativa em um tom mais escuro. As colaborações mais significativas estão ente o Reino Unido e Austrália e Reino Unido e Nova Zelândia, Austrália e Nova Zelândia, Estados Unidos e China e, ainda que com baixa produção sobre a temática, Uganda e o Kênya. 
Figura 2. Mapa mundial com as ligações de colaboração interpaíses.

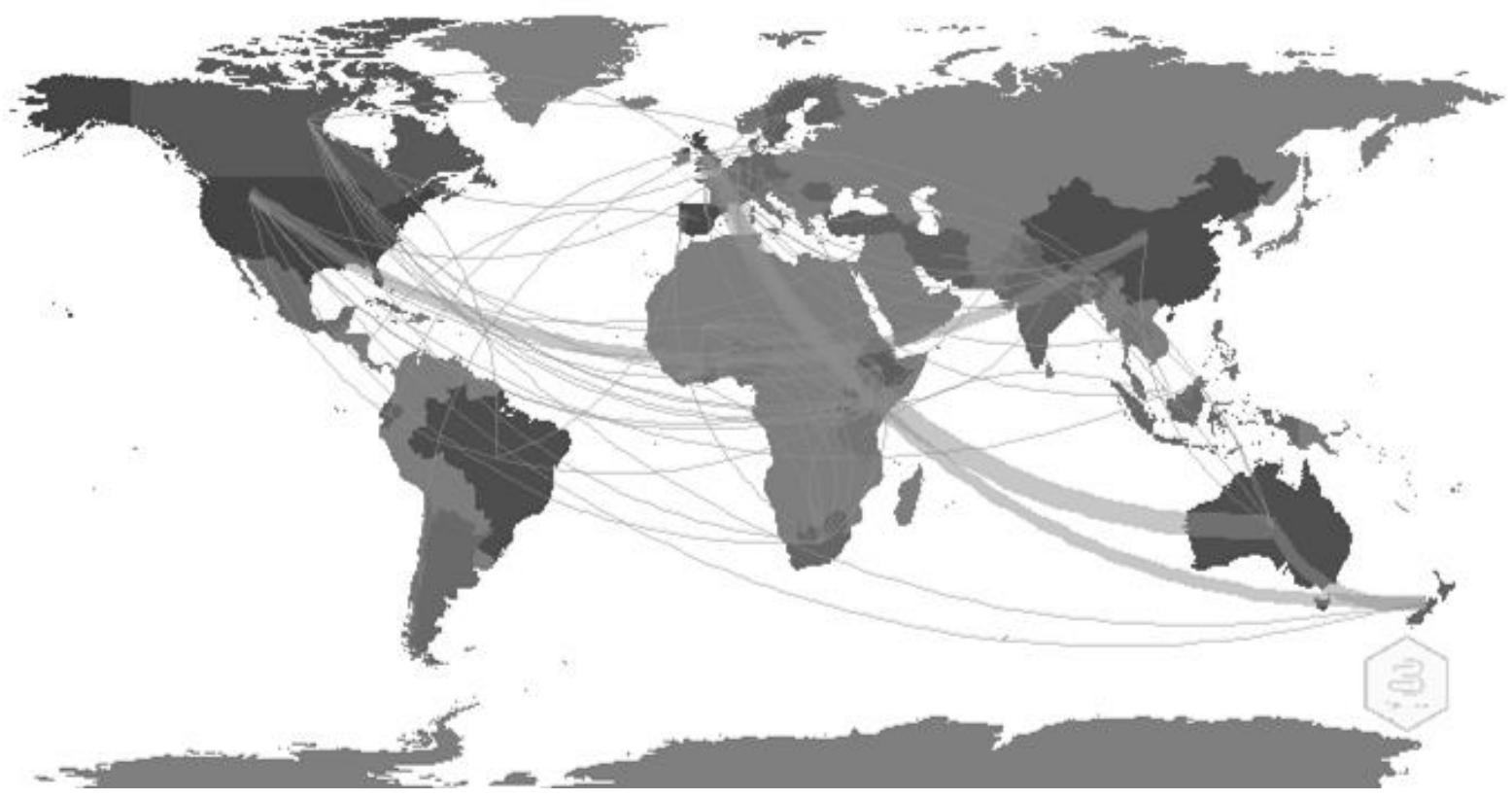

Fonte: Autores (2022).

Outra percepção possível, a partir da Figura 2 e seu detalhamento no Quadro 1, é que os colaboradores não demonstraram uma relação mais continental, interagindo com os países mais próximos, como por exemplo Brasil/EUA, França/Reino Unido, ainda que a Austrália/Nova Zelândia apareçam neste contexto. Há uma conexão percebida na produção do Canadá, particularmente com países do continente africano, da Austrália e Brasil com países de culturas bem distintas e não regionalizadas. Esta conexão canadense pode sugerir que na produção desta temática de educação em saúde e idosos há uma aproximação de pesquisa, que podem ter sido realizadas no continente africano, ou com resultados comuns entre os pesquisadores.

O Quadro 1 demonstra uma maior aproximação entre os pesquisadores do Reino Unido e da Austrália, do Reino Unido com a Nova Zelândia e dos Estados Unidos com a China, com uma alta frequência de relacionamentos. O continente africano conta com muitas interações entre seus pesquisadores, sendo que Uganda e Quênia possuem relacionamentos muito importantes nesta área de estudo. 
Quadro 1. Origem, destino e frequência das principais relações de colaboração entre autores interpaíses.

\begin{tabular}{|l|l|c|}
\hline Origem & Destino & Frequência \\
\hline United Kingdom & Australia & 4 \\
\hline Australia & New Zealand & 2 \\
\hline Uganda & Kenya & 2 \\
\hline United Kingdom & New Zealand & 2 \\
\hline Usa & China & 1 \\
\hline Australia & Canada & 1 \\
\hline Australia & Denmark & 1 \\
\hline Australia & Iran & 1 \\
\hline Brazil & Canada & 1 \\
\hline Brazil & Pakistan & 1 \\
\hline Brazil & Portugal & 1 \\
\hline Canada & Congo & 1 \\
\hline Canada & Denmark & 1 \\
\hline Canada & Ethiopia & 1 \\
\hline Canada & Kenya & 1 \\
\hline Canada & Liberia & 1 \\
\hline Canada & Mali & 1 \\
\hline Canada & New Zealand & \\
\hline Canada & Rwanda & 1 \\
\hline & & 1 \\
\hline
\end{tabular}

Fonte: Autores (2022).

Ainda que a participação de estrangeiros nas pesquisas brasileiras seja insipiente, é possível perceber que os principais parceiros externos são o Canadá, o Paquistão e Portugal, com uma coautoria cada, sendo o Canadá um dos importantes players neste processo de internacionalização. Pode-se inferir que o incremento de pesquisadores internacionais na coautoria de estudos brasileiros pode ajudar a melhorar a produção científica nacional sobre o tema de educação em saúde para idosos, dar maior abrangência e visibilidade, ou contar com fontes de financiamentos externas.

\subsection{Lei de Bradford (sources)}

No título anterior foi possível perceber a dispersão e a colaboração de autores, além de sua produtividade absoluta e relativa. Neste momento será possível avaliar a dispersão temática das revistas (sources), seu núcleo contextual e a sua produtividade, estimando o grau de relevância do periódico em relação ao tema. A lei de Bradford, criada em 1934, busca estabelecer o núcleo de periódicos sobe um assunto específico em relação ao contexto periférico deste assunto, considerando variáveis de aproximação (Silva \& Mayworm, 2020).

A lei de Bradford possibilita ainda um zoneamento por produtividade dos periódicos, indicando assim que as fontes mais concentradoras de um determinado tema são consideradas como pertencentes à zona 'core' (núcleo) daquele tema em um gráfico por produtividade, e situar as demais fontes como centro (produção mediana) e periféricas para aquelas com pequena produtividade, considerando o tema e o período pesquisado (Gregorio-Chaviano et al., 2020).

Neste sentido, há uma preocupação de se catalogar ou indexar as fontes segundo o espaço de publicação proporcionado a assuntos que não são considerados escopo principal das revistas. Segundo Silva et al., (2020, p.40), a Lei de Bradford “tem o objetivo de descobrir a extensão na qual artigos de um assunto científico específico apareciam em periódicos destinados a outros assuntos, estudando a distribuição dos artigos em termos de variáveis de proximidade ou de afastamento" 
Os autores Sembay et al. (2020, p. 2) afirmam também que segundo a Lei de Bradford, a "sua representação é realizada por zonas, e sua distribuição é apresentada em três zonas, sendo todas representadas por 33\% das publicações".

A Figura 3 apresenta a distribuição dos periódicos coletados nesta revisão, por zoneamento segundo a Lei de Bradford, com três zonas. A zona 1, zona núcleo ou core zone, apresentou 5 periódicos, com uma frequência de publicações de 118 artigos. A zona 2 demonstrou um afastamento entre o número observado, que foram 34 periódicos e o número esperado pela equação de Bradford, em que eram estimados 25 periódicos. Por fim, houve uma inversão de proporções entre o observado na zona 3, 102 periódicos e o projetado, 125 periódicos. Estes dados quanto ao número de periódicos não afetam a constante de Bradford para a produtividade por zona, que foi de 0,976 (proporção 1:n:n²) (Sembay et al., 2020).

Figura 3. Gráfico representativo da dispersão dos periódicos, segundo a equação de Bradford.

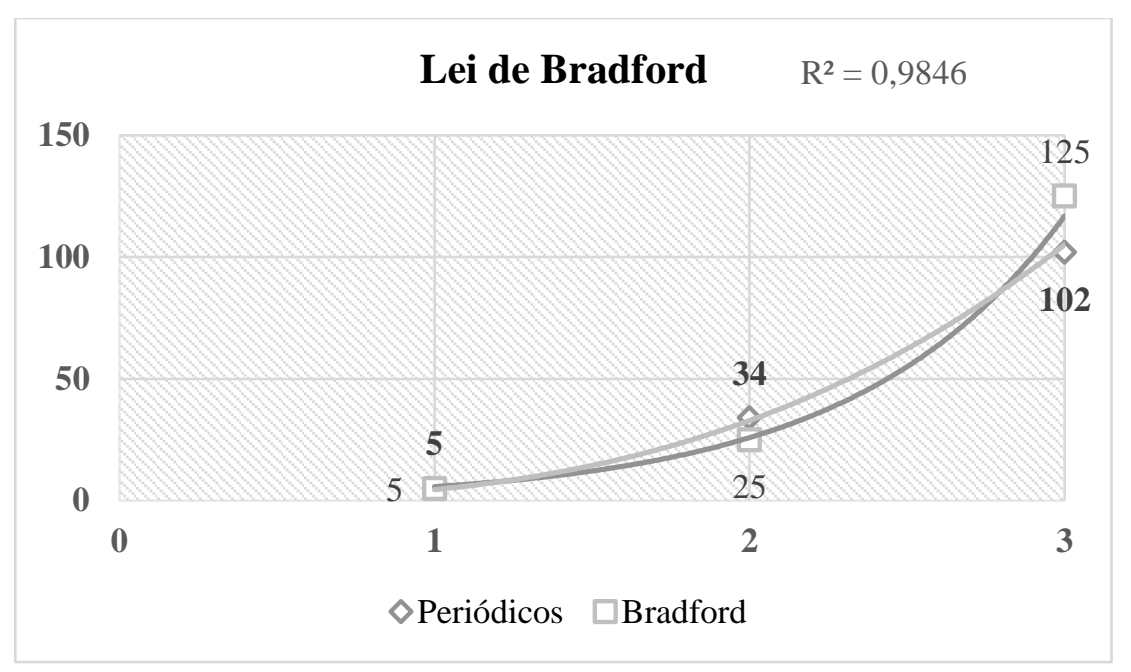

Fonte: Autores (2022).

A Figura 4 representou graficamente a distribuição por produtividade das revistas que mais publicam sobre o tema Educação em saúde e idosos, sendo que as 5 revistas mais produtivas foram concentradas na zona 1 (core) do gráfico. O ranking desta distribuição é apesentado no Quadro 2 considerando a frequência (decrescente), zona e indicadores de produtividade dos periódicos. A Bibliometria proporciona a compreensão de quais periódicos são mais focados em determinado tipo de conhecimento, facilitando assim a disseminação da ciência. Os pesquisadores de determinado tema podem reduzir suas buscas por periódicos quando possuem uma cartografia que lhes indique a concentração de publicações na área.

No caso das publicações sobre educação em saúde para idosos, as revistas dos países de língua inglesa são prevalentes, conforme demonstrado no Quadro 2. Ainda que os Estados Unidos possua o periódico com maior frequência de publicações, o Reino Unido contribui com um maior número de revistas, que somadas as suas publicações, ultrapassam os Estados Unidos em quantidade publicada no período.

Segundo Silva, Almeida e Grácio (2018, p. 133), há que se avaliar a produtividade e efetividade dos periódicos sobre um determinado tema, a fim de poder extrair um grupo selecionado quanto À qualidade de suas publicações. Neste caso, “os indicadores bibliométricos vêm ocupando um papel relevante no processo avaliativo da produção científica e, entre eles, destacam-se o Fator de Impacto (FI) e o Índice h". O índice H (H-index) é um importante instrumento de medida do impacto científico, visto que ele mede uma combinação de fatores entre produtividade e a quantidade de citações. 
Figura 4. Gráfico de zoneamento de periódicos sobre o tema Educação em saúde e Idosos, considerando a quantidade de artigos produzidos por periódico.

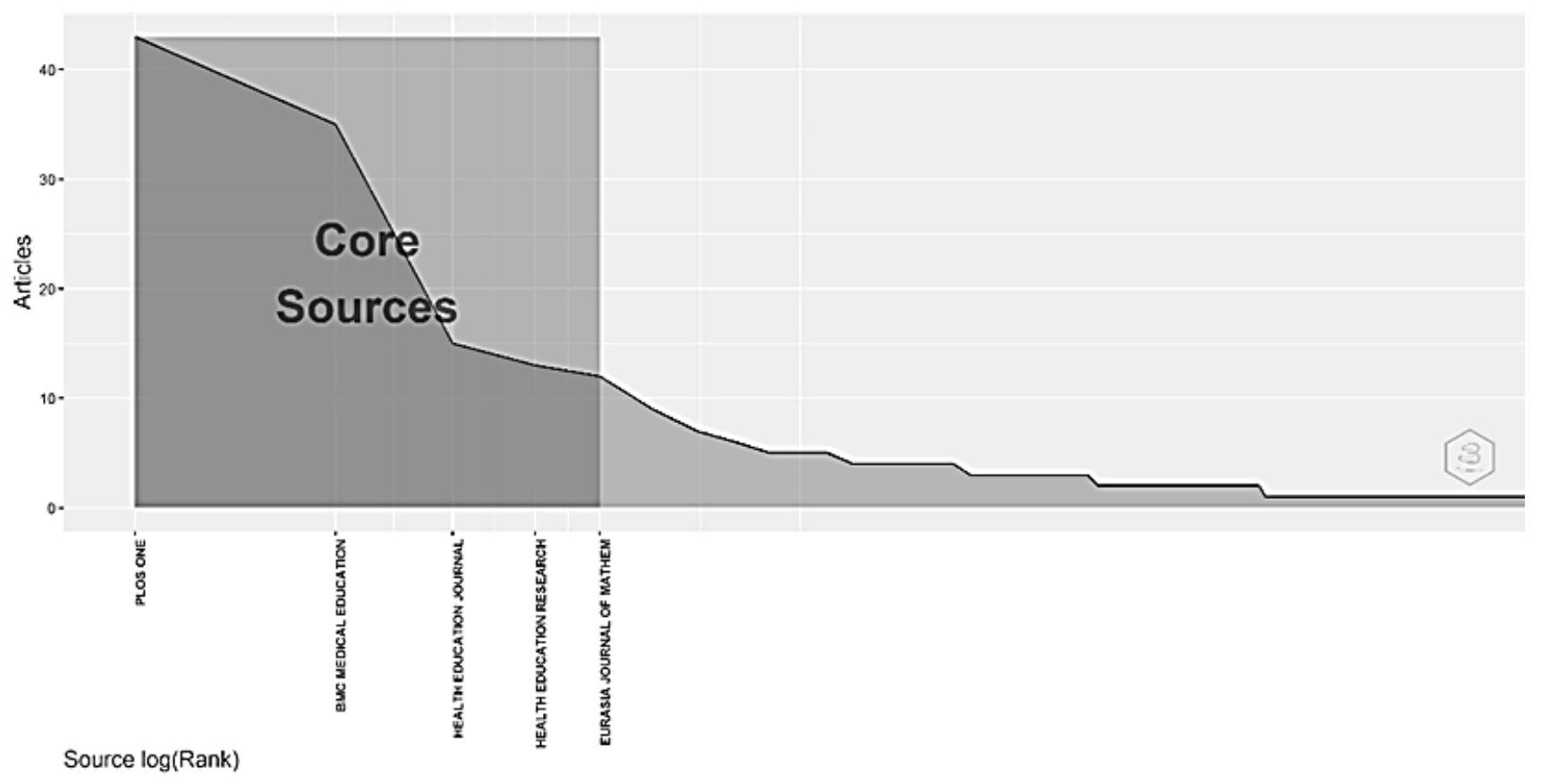

Fonte: Autores (2022).

O Quadro 2 confirma como principal periódico a revista Plos ONE, com uma frequência de 43 publicações. Tanto na produtividade medida pelo Scimago Journal \& Country Rank ${ }^{2}$ (SJR), como pelo H-index, a Plos ONE apresenta uma diferença significativa para o segundo lugar do ranking, a BMC Medical Education. As 5 primeiras revistas compõem a zona principal (core) do mapeamento de revistas sobre a temática. Há de se notar que os Estados Unidos terem uma boa produtividade, apenas 1 revista está na zona principal, enquanto o Reino Unido participa com 3 periódicos. Os indicadores de produtividade foram extraídos do SJR e levam em consideração os indicadores de 2020.

Quando verificada a frequência absoluta de publicações, nota-se que as revistas do núcleo central possuem acima de 10 publicações sobre o tema no período pesquisado e não consta nenhuma revista brasileira no contexto das 10 principais revistas (núcleo e zona central). Há uma percepção de concentração das revistas em seu escopo principal e a falta de revistas especializadas no tema.

\footnotetext{
2 SCImago Journal \& Country Rank é um portal disponível ao público que inclui periódicos e indicadores científicos do país desenvolvidos a partir das informações contidas na base de dados Scopus® (Elsevier BV) e está disponível em https://www.scimagojr.com/
} 
Quadro 2. Ranking das 10 principais revistas, frequência absoluta de publicações, zoneamento por importância, e indicadores de produtividade SJR e H-index.

\begin{tabular}{|c|c|c|c|c|c|c|}
\hline Rank & Periódico & País & Freq. & Zona & SJR & H-index \\
\hline 1 & Plos One & USA & 43 & Zone 1 & 0,99 & 332 \\
\hline 2 & Bmc Medical Education & UK & 35 & Zone 1 & 0,81 & 68 \\
\hline 3 & Health Education Journal & UK & 15 & Zone 1 & 0,42 & 34 \\
\hline 4 & Health Education Research & UK & 13 & Zone 1 & 0,60 & 103 \\
\hline 5 & Eurasia Journal of Mathematics Science And & Turkey & 12 & Zone 1 & 0,44 & 38 \\
\hline 6 & Technology Education & USA & 9 & Zone 2 & 0,85 & 86 \\
\hline 7 & Journal of School Health & Switzerland & 7 & Zone 2 & 0,45 & 19 \\
\hline 8 & Education Sciences & UK & 6 & Zone 2 & 0,00 & 53 \\
\hline 9 & Jocedia - Social And Behavioral Sciences & USA & 5 & Zone 2 & 0,71 & 95 \\
\hline 10 & Revista Complutense De Educación & SPAIN & 5 & Zone 2 & 0,47 & 14 \\
\hline
\end{tabular}

Nota. Freq.: Frequência (quantidade de publicações do periódico); SJR : Scientific Journal Ranking; H-index:índice H. Fonte: Autores (2022).

A Tabela 6 elenca as 10 principais instituições de pesquisa, sendo que apenas 1 delas não se trata de uma universidade, o que pode sugerir que a pesquisa e o conhecimento gerado tenham maior financiamento no meio acadêmico. As instituições brasileiras não estão entre as 10 principais fomentadoras de estudos na área, ainda que a projeção de crescimento da população idosa seja importante para 2050 e que os custos com a saúde pública possam crescer desproporcionalmente neste período.

Segundo o site da Johns Hopkins Medicine International (JHMI) no Brasil, em 2013, esta instituição recebeu a afiliação do Hospital Moinhos de Vento, com sede em Porto Alegre/RS. A JHMI, que é considerada a melhor empresa em saúde dos Estados Unidos, conforme avaliação da News and World Report (empresa de análise e classificação empresarial) apresenta como sendo o seu principal objetivo: promover a “disseminação global da missão da Johns Hopkins Medicine: desenvolver a saúde da comunidade local e mundial por meio da excelência em educação médica, pesquisa e assistência” (Jhmi, 2021, p. online).

Tabela 6. Produção científica sobre educação em saúde e idosos, por instituição de pesquisa, 10 principais em ordem decrescente.

\begin{tabular}{ll}
\hline INSTITUIÇÃO & PUBLICAÇÕES \\
\hline Johns Hopkins University & 9 \\
Auckland University Technology & 8 \\
Orebro University & 8 \\
Palacky University Olomouc & 8 \\
University Pablo de Olavide & 8 \\
University Sydney & 8 \\
Emory University & 7 \\
Hacettepe University & 7 \\
Makerere University & 7 \\
Mayo Clinic & 7 \\
\hline
\end{tabular}

Fonte: Autores (2022).

O Hospital Moinhos de Vento possui uma expressiva área de educação, pesquisa e desenvolvimento, que atende a cursos em nível de graduação e pós-graduação, cursos técnicos e de extensão universitária e que pode ser considerado como a representação brasileira neste ranking. 


\subsection{Lei de Zipf (keywords)}

A Lei de Zipf considera a coocorrência (frequência) de descritores e palavras-chave nas publicações e entende que há um padrão que emerge nas publicações, uma tendência de baixa dispersão quando se estabelece um determinado tema, ou seja, os autores consideram um mesmo grupo de descritores para definir um determinado fenômeno. Neste caso, poucos descritores serão usados muitas vezes para o tema estudado (Beira et al., 2020).

A equação desta teoria é explicada pela fórmula $y=f(1 / x)$, onde $y$ é o valor esperado pela lei de Zipf, o $f$ representa a frequência em que a palavra aparece nos artigos e $x$ é a posição desta palavra no ranking de descritores.

$\mathrm{Na}$ extração de dados realizada pelo software, foram coletados 977 descritores (keywords), sendo que os 10 principais descritores estão relacionados na tabela 7. A expressão health education (educação em saúde) aparece em primeiro lugar dentre os descritores.

O site de Descritores em Ciências da Saúde (DeCS) define, como escopo, que este termo tem por objetivo "desenvolver nas pessoas um sentido de responsabilidade, como indivíduo, membro de uma família e de uma comunidade, para com a saúde, tanto individual como coletivamente" (Bireme, 2021, p. online). Este descritor foi criado em 1966 e revisado em 2015 pelo DeCS e, desde sua criação, foram cadastrados na Biblioteca Virtual em Saúde (BVS) um total de 48914 artigos até 2021, abordando os mais diversos assuntos, participantes e contextos, sendo que destes, 30794 artigos foram redigidos na língua inglesa e 7794 publicações no idioma português (Bvs, 2021).

Tabela 7. Principais descritores, considerando os 10 mais citados no período de 2017 a 2021, nas bases Scopus e Web of Science.

\begin{tabular}{ll}
\hline $\begin{array}{l}\text { DESCRITORES POR FREQUÊNCIA ABSOLUTA } \\
\text { (10 principais) }\end{array}$ & Ocorrências \\
\hline Descritores & 91 \\
\hline Health Education & 32 \\
Education & 18 \\
Health & 10 \\
Nutrition & 10 \\
Physical Education & 8 \\
Curriculum & 8 \\
Medical Education & 6 \\
Children & 6 \\
Health Literacy & 6 \\
Health Promotion & \\
\hline
\end{tabular}

Fonte: Autores (2022).

Como previsto pela lei de Zipf, os descritores mais relevantes para circunscrever o tema de educação em saúde para idosos são o próprio descritor 'Educação em saúde' com 91 ocorrências, 'Educação' com 32 coocorrências e 'Saúde' com uma frequência de 18 ocorrências, o que é interessante, pois segundo a equação de Zipf (Figura 4), o número esperado para o descritor education deveria ser 46 e não 32, que representaria a terceira posição ranking. Este resultado pode inferir que há um descritor oculto não registrado na coleta dos dados. 
Research, Society and Development, v. 11, n. 3, e43911326884, 2022

(CC BY 4.0) | ISSN 2525-3409 | DOI: http://dx.doi.org/10.33448/rsd-v11i3.26884

Figura 5. Distribuição dos descritores por frequência observada e o esperado pela equação de Zipf.

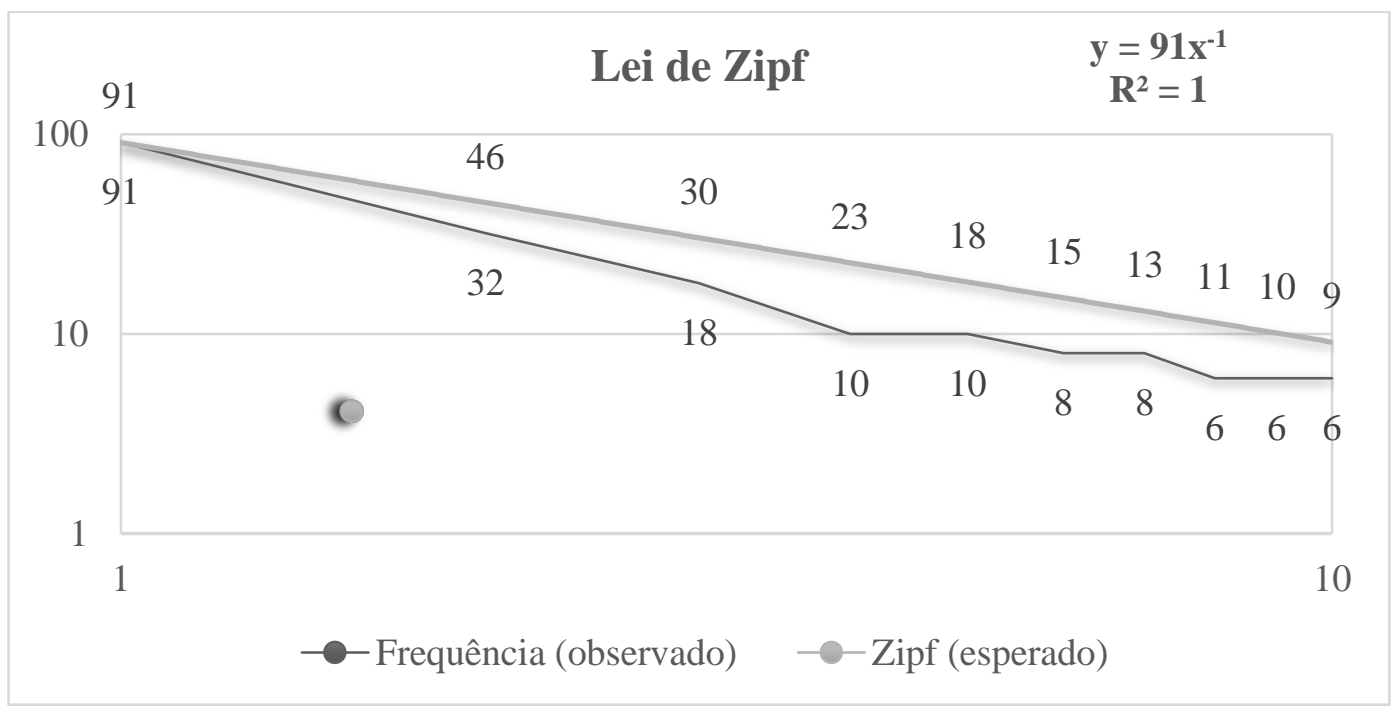

Fonte: Autores (2022).

Percebe-se que entre os 10 principais não estão citados os descritores relativos ao tema idosos, mas sim 'crianças' (children), que segundo o DeCS referem-se à pessoas “de 6 a 12 anos de idade. Um indivíduo de 2 até 5 anos de idade é PRÉESCOLAR” (Bireme, 2021). Ainda percebe-se que há uma consistência nos estudos com outras faixas etárias, ainda que os estudos abordem de alguma forma a idade idosa.

Figura 6. Evolução e estratificação temática no período 2017-2021.

2017-2019

2020-2021

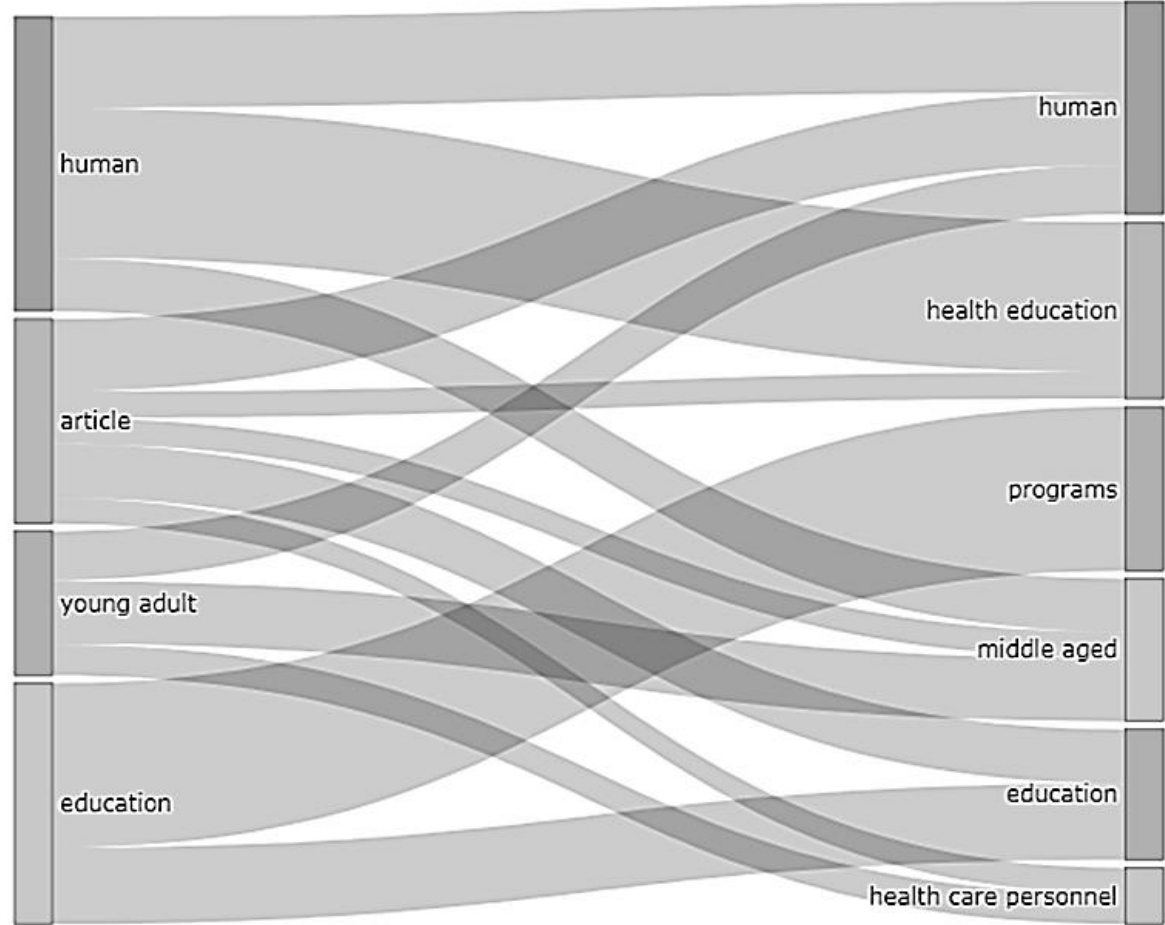

Fonte: Autores (2022). 
A Figura 6 apresenta, de forma visual, a mudança evolutiva nos temas relacionados à educação em saúde. Percebe-se, em primeiro plano, a estratificação dos temas, que neste caso, em 2017 haviam 4 temas principais que, ao longo de 5 anos, tornaram-se 6 temas.

Nesta complexidade apresentada pela evolução temática, aparece uma tecitura que integra significados e especifica caminhos que antes estavam à margem nas publicações. Na representação, o que antes era tratado como próprio do ser humano, recebeu temas adicionais, como a definição de grupos de meia idade. Em 2017 ainda falava-se muito em jovens adultos e é possível que o aumento da população idosa e o crescimento da pirâmide etária, com a estabilização das camadas mais jovens, tenha levado os pesquisadores a ampliar os estudos para a população mais madura.

O tema educação também bifurcou-se ao longo do tempo, sendo visto também pela perspectiva das políticas públicas e pelos métodos de ensino/aprendizagem que se relacionam ao tema 'programas'. Há um grande significado neste quesito, uma vez que o descritor demonstra que o tema educação em saúde saiu do campo das ideias, partindo para aplicações concretas na práxis educativa.

O quadro 3 demonstra o crescimento real dos descritores mais usados para definir o tema educação em saúde. O descritor ‘educação em saúde' cresceu, nos últimos 5 anos, a uma taxa de 404,54\% sobre o resultado de 2017, o que demonstra que a percepção dos pesquisadores no tema é relativamente importante, ainda que os números mínimos e máximos seja pequenos. Aqui não foram computados os indicadores que representam os idosos, devido ao tema não ser registrado com muita frequência entre os descritores.

Quadro 3. Evolução da importância do descritor ao longo do período pesquisado.

\begin{tabular}{|l|c|c|c|c|c|}
\cline { 2 - 6 } \multicolumn{1}{c|}{} & \multicolumn{5}{c|}{ Ano } \\
\hline Descritores & 2017 & 2018 & 2019 & 2020 & 2021 \\
\hline Health Education & 22 & 36 & 59 & 80 & 89 \\
\hline Education & 5 & 12 & 22 & 28 & 32 \\
\hline Health & 3 & 5 & 10 & 12 & 18 \\
\hline Nutrition & 2 & 4 & 8 & 8 & 10 \\
\hline Physical Education & 1 & 3 & 5 & 6 & 9 \\
\hline Curriculum & 2 & 3 & 5 & 6 & 8 \\
\hline Medical Education & 1 & 3 & 4 & 7 & 8 \\
\hline
\end{tabular}

Fonte: Autores (2022).

A Figura 7 apresenta as conexões entre os descritores, por comunidades de sentido. A distribuição dos descritores segue uma construção matemática que define a força das conexões, segundo o grau de proximidade entre os mesmos e a largura da linha. Também foi possível perceber as ligações entre os núcleos centrais de cada comunidade e as relações menores periféricas. A dimensão do círculo também está relacionada à importância relativa do descritor na amostra coletada.

Nesta distribuição é possível notar que a educação em saúde é a maior coocorrência entre os descritores e também a que mais demanda interligações diversas com os demais descritores. O grafo possibilita também perceber que alguns estudos tratam com maior frequência dos descritores saúde e educação, de forma separada. Também é verificada a forte relação que existe entre o descritor educação em saúde e a educação física, o que pode ser um caminho para inclusão de práticas voltada para o ensino de saúde para os idosos, seja por meio de programas formais, como também informais, como nas academias de ginástica e, até mesmo nas atividades não formais, exercidas por profissionais em espaços públicos, como parques.

Estas ligações são representativas para o tema analisado, de forma a fornecer tanto estímulos para a educação em saúde nos idosos, como para profissionais e instituições. Um estudo sobre a percepção de saúde de idosas participantes de um programa 
de educação demonstrou que a percepção de saúde envolve fatores externos (educação e ambiente) e internos (aprendizagem e autoconsciência) ao idoso e que a saúde não está relacionado apenas a ausência de doença, o que apoia a ideia de que a educação em saúde ajuda no bem estar e na qualidade de vida do idoso (Cabral et al., 2020).

Figura 7. Análise da rede de conexões entre os descritores.

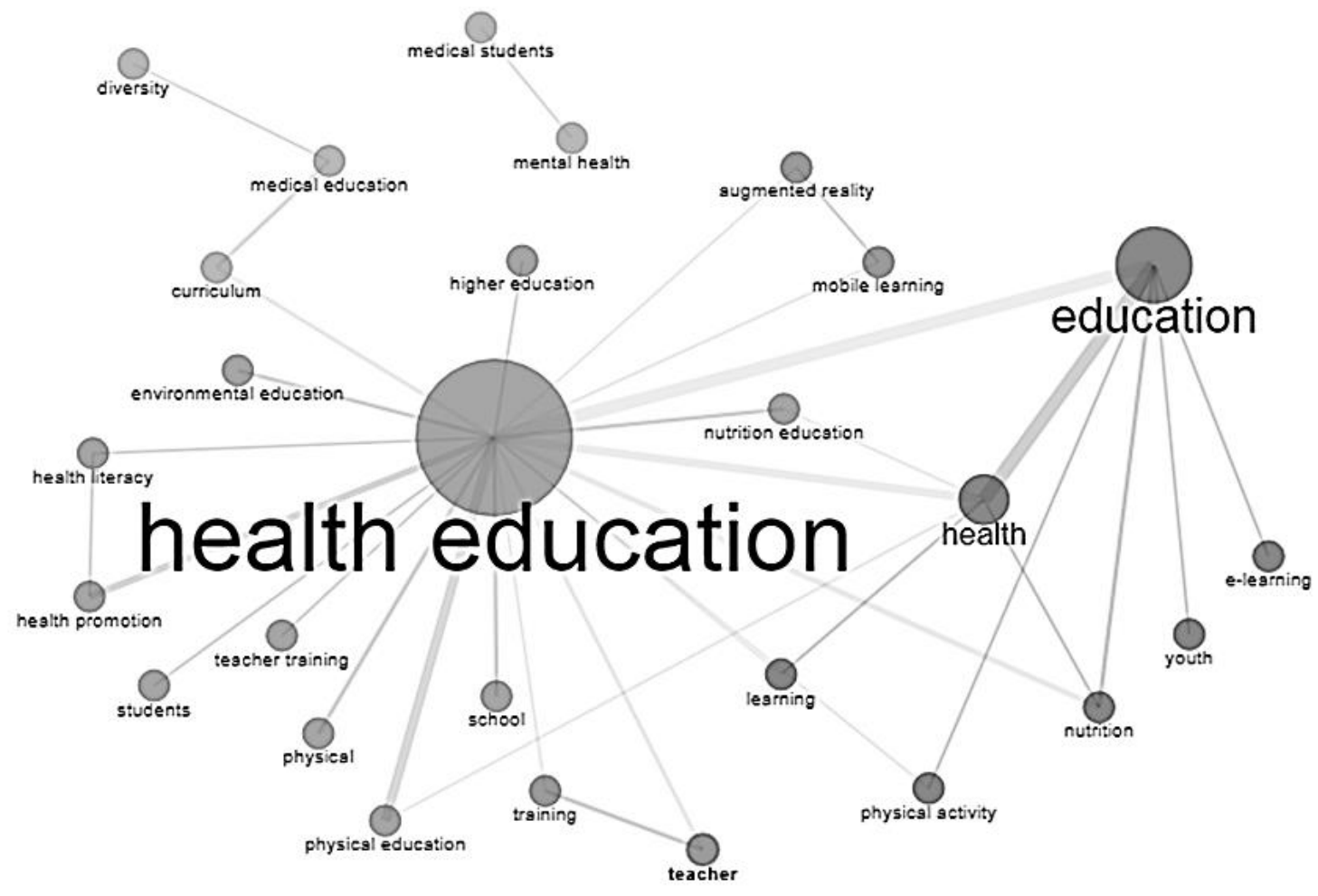

Fonte: Autores (2022).

Percebe-se também a gama de outros temas relevantes ligados à educação em saúde, como por exemplo o treinamento de educadores, o próprio ambiente educacional, a escola, a educação superior e o currículo. Também são vistas as novas tecnologias da informação, com os aplicativos de aprendizado móvel, disponíveis em smartphones e tablets, como a realidade aumentada, na produção de conteúdo ligado à nutrição e boas práticas de saúde e bem estar. Nesta visualização não foi possível verificar descritores com força relevante para a idade idosa.

\section{Considerações Finais}

Este estudo bibliométrico foi elaborado a partir da questão 'como se apresentou a produção científica sobre o tema Educação em Saúde para Idosos entre os anos de 2017 e 2021?’ e foi sendo analisado e discutido até que foi possível realizar o objetivo geral deste estudo, que foi 'apresentar o perfil da produção científica sobre os temas Educação em Saúde ligado à população idosa', o que foi construído em etapas definidas, a priori, no planejamento do mesmo, a partir das análises da rede de colaboradores com os critérios da lei de Lotka, da dispersão dos documentos com o uso da lei de Bradford e, da frequência de descritores utilizados nas publicações, conforme a lei de Zipf.

O perfil da produção científica ainda é pouco expressivo mundialmente, sendo que a média de intervalo das publicações gira em torno de 2 anos, além da média de citações por ano destes documentos ser menor que 1 citação.

Também foram encontradas poucas revisões (19) sobre o assunto nas bases de dados consultadas, confirmando a baixa produtividade do tema. Foi também interessante perceber que os países com uma população mais idosa, como o Japão, a Itália, 
a Grécia, a Alemanha e Portugal não aparecerem nos rankings de publicações, caso que deve ser melhor avaliado em revisões sistemáticas, seja para entender este comportamento ou para descobrir os métodos aplicados por estes países para resolver os problemas relacionadas ao tema.

Neste sentido, coube investigar os achados a partir das leis clássicas da Bibliometria, o que apresentou alguns resultados interessantes. Por exemplo, a média de coautoria na produção sobre o tema é de 4,38 autores por documento, o que leva a reflexão de que sejam grupos de pesquisa voltados para a questão da educação em saúde para idosos. Na amostra, apenas 3 autores realizaram 4 estudos, enquanto que a grande maioria (96\% ou 1328 autores) produziu apenas um documento sobre o tema. O Brasil figura em $5^{\circ}$ lugar na produção autoral sobre o tema, com mínima participação de autores estrangeiros (4\%), enquanto países como o Canadá e o Reino Unido dependem de maior participação externa neste tipo de assunto. Esta autonomia do Brasil no tema, demonstra um maior esforço dos grupos de pesquisa brasileiros na busca de soluções e compreensão do fenômeno do envelhecimento e da educação em saúde.

Mesmo com a posição brasileira no ranking de autores, as revistas utilizadas para a publicação foram as estrangeiras, em especial, a americana Plos ONE. As revistas do Reino Unido conquistaram 3 posições entre as 5 maiores, o que torna este país um relevante celeiro de publicações sobre o tema. Nenhuma revista brasileira constou entre as 10 maiores do ranking, o que causa um desencaixe entre a produção científica e a publicação dos estudos brasileiros e revela ainda uma questão a ser desenvolvida, a autonomia brasileira em publicações. As revistas brasileiras, para a amostra deste estudo, não apresentaram escopo para tal tema, o que pode criar maiores dificuldades para os autores nacionais.

Outra questão percebida foi o fato do financiamento de pesquisa, que está majoritariamente nas mãos de universidades, o que demonstra ainda uma falta de interesse pelo assunto a partir de governos, inclusive nesta amostra, somente apareceu uma instituição privada no financiamento de pesquisas. Os governos ainda não perceberam a importância do assunto, seja a nível econômico, como a nível social, que melhoraria o indicador de desenvolvimento humano com consequentes implicações nas demais áreas nacionais.

$\mathrm{Na}$ análise por descritores, o que mais preocupa é o fato de que os idosos ainda não estão devidamente representados, sendo que aparecem como temas coadjuvantes no processo de análise do tema educação em saúde, fato que ocorre com mais frequência na população infantil. Ainda assim, percebe-se uma evolução do tema ao longo destes últimos 5 anos, com uma mudança na faixa etária, o que é significativo para alcançar a população da idade idosa, partindo de 'adultos jovens' para o descritor 'meia idade', sendo que este movimento segue o processo de produção sobre o tema. Além disso, percebe-se que a educação em saúde é um tema que obteve um maior fluxo produtivo, talvez tenha sido, em parte, catalisada por força da pandemia de Covid-19, mas observa-se que existem grupos de estudo voltados para o fenômeno do envelhecimento, incentivados pelo crescimento desta população idosa em relação às demais faixas etárias.

Por fim, diversas lacunas estão abertas para o desenvolvimento de pesquisas mais aprofundadas, especialmente no Brasil, sendo um campo aberto para a produção científica.

\section{Referências}

Araújo, C. A. (2006). Bibliometria: evolução histórica e questões atuais. Em Questão, 12(1), 11-32. https://doi.org/https://doi.org/10.22478/ufpb.1981$0695.2007 \mathrm{v} 2 \mathrm{n} 1.8023$

Aria, M., \& Cuccurullo, C. (2021). Package bibliometrix: Comprehensive Science Mapping Analysis. Manual, Bibliometrix. https://cran.rproject.org/web/packages/bibliometrix/bibliometrix.pdf

Beira, J. C., Gontijo, M., Santa Anna, J., \& Maculan, B. (2020). INDICADORES BIBLIOMÉTRICOS NA PRODUÇÃO CIENTÍFICA EM PERIÓDICOS BRASILEIROS DA CIÊNCIA DA INFORMAÇÃO. Revista ACB: Biblioteconomia em Santa Catarina, $25(2)$, 383-408. https://revista.acbsc.org.br/racb/article/view/1660 
Research, Society and Development, v. 11, n. 3, e43911326884, 2022

(CC BY 4.0) | ISSN 2525-3409 | DOI: http://dx.doi.org/10.33448/rsd-v11i3.26884

Bireme. (2021). DeCS - Descritores em Ciências da Saúde. Retrieved 25 de Julho de 2021, from DeCS/MeSH Descritores em Ciências da Saúde: https://decs.bvsalud.org/

Brasil, M. d. (2021). Glossário Eletrônico: Terminologia da Saúde: BVS Ministério da Saúde. Retrieved 20 de Julho de 2021 , from Biblioteca Virtual em Saúde. Ministério da Saúde: http://bvsms2.saude.gov.br/php/level.php?lang=pt\&component=44\&item=114

Bvs. (2021). Pesquisa | Portal Regional da BVS. Retrieved 10 de Dez. de 2021, from bvs - biblioteca virtual em saúde: https://pesquisa.bvsalud.org/portal/

Cabral, M. A., Flores-Gomes, G., Arruda, M. L., Beggiato, S. M., Oliveira, V. d., \& Vagetti, G. C. (2020). Perceção de Saúde de Idosos Participantes de um Programa de Inclusão Digital. Revista Brasileira De Ciências Da Saúde, 1-12. https://doi.org/https://doi.org/10.22478/ufpb.2317-6032.2020v24n4.53136

Cope. (1999). Guidelines on good publication practice. Committee on Publication Ethics (COPE), [online]. Retrieved 21 de Agosto de 2020, from https://publicationethics.org/files/u7141/1999pdf13.pdf

Flores-Gomes, G., Lopes, R. F., Oliveira, V. d., \& Vagetti, G. C. (2021). Educação em Saúde para Idosos: um protocolo de revisão bibliométrica da produção científica de 2017 a 2021. Curitiba. https://doi.org/http://dx.doi.org/10.17605/OSF.IO/BF9P8

Gama, J. C., Neto, A. F., \& Santos, W. d. (2021). Formação para atuação com o esporte: características bibliométricas e redes de colaboração. Movimento, 27(e27017), 1-18. https://doi.org/https://doi.org/10.22456/1982-8918.109936

Gregorio-Chaviano, O., Limaymanta, C. H., \& López-Mesa, E. K. (2020). Análisis bibliométrico de la producción científica latinoamericana sobre COVID-19. Biomédica, 40(2), 104-115. https://doi.org/http://dx.doi.org/10.7705/biomedica.5571

Jhmi. (2021). JOHNS HOPKINS MEDICINE INTERNATIONAL - Hospital Moinhos de Vento. from Hospital Moinhos de Vento: https://www.hospitalmoinhos.org.br/institucional/o-hospital/johns-hopkins-medicine-internacional

Mugnaini, R., Fujino, A., \& Kobashi, N. Y. (2017). Bibliometria e cientometria no Brasil: infraestrutura para avaliação da pesquisa científica na era do Big Data. São Paulo , SP, Brasil: ECA/USP. https://doi.org/https://dx.doi/10.11606/9788572051705

Pérez-Escoda, A. (2017). WOS e SCOPUS: Os grandes aliados de todo pesquisador. Comunicar. https://doi.org/https://doi.org/10.3916/escola-de-autores-031 Rstudio. (2022). RStudio- Open Source \& professional software for data. RStudio: https://www.rstudio.com/

Saldiva, P. H., \& Veras, M. (2018). Gastos públicos com saúde: breve histórico, situação atual e perspectivas futuras. ESTUDOS AVANÇADOS, 32(92), 47-61. https://doi.org/http://dx.doi.org/10.5935/0103-4014.20180005

Sembay, M., Pinto, A., \& Macedo, D. y. (2020). Aplicación de la Ley de Bradford a la investigación sobre Open Government. Anales de Documentación, 23(1). https://doi.org/http://dx.doi.org/10.6018/analesdoc.326771

Silva, D. D., Almeida, C., \& Grácio, M. (2018). Associação do Fator de Impacto e do Índice h para a avaliação de periódicos científicos: uma aplicação no campo da Ciência da Informação. Em Questão, 24, 134-151. https://doi.org/https://doi.org/10.19132/1808-5245240.132-151

Silva, V. R., \& Mayworm, P. (2020). Indicadores bibliométricos de colaboração científica na área de enfermagem do trabaho. Rev. Eletr de Cien. Tecnol e Inova, 10, 36-54. https://doi.org/http://dx.doi.org/10.9789

Soares, S. V., Picolli, I. R., \& Casagrande, J. L. (2018). Pesquisa Bibliográfica, Pesquisa Bibliométrica, Artigo de Revisão e Ensaio Teórico em Administração e Contabilidade. Administração: Ensino E Pesquisa, 19(2), 308-339. https://doi.org/https://doi.org/10.13058/raep.2018.v19n2.970

Vagetti, G. C., Hackenberg, C., Flores-Gomes, G., Arruda, M., Beggiato, S., \& Oliveira, V. (2020). Políticas públicas em saúde, violência, educação e assistência social para pessoas idosas. Research, Society and Development, 9(8), 1-21. https://doi.org/DOI: http://dx.doi.org/10.33448/rsd-v9i8.5868 